\title{
Teoría de la justicia de Rawls. Una crítica-matemática a la crítica de Amartya Sen a John Rawls
}

\author{
Gardy Augusto Bolívar Espinoza \\ Universidad Autónoma de México-Azcapotzalco. \\ Email: abe88@hotmail.com
}

\section{Oscar Rogelio Caloca Osorio}

Universidad Autónoma de México-Azcapotzalco.

Email: oscarcalo8@yahoo.com.mx

\begin{abstract}
Resumen: El artículo se encuadra en el análisis a la Teoría de la Justicia de Rawls desde diferentes perspectivas. En este caso, refiere a: (1) la crítica de Amartya Sen a algunos aspectos del modelo de Rawls; y, (2) a la respuesta a estos argumentos desde la metateoría estructuralista y la matemática de los juegos. Sostenemos que la postura de Sen se debilita al cambiar las condiciones iníciales del modelo de Rawls y por los magros resultados matemáticos de la corrección propuesta por Sen. El resultado es que el modelo de Rawls no se arregla por la crítica de Sen y obliga a considerarlo desde una perspectiva ideal normativa. En esta perspectiva no se desecha el cálculo matemático, que es esencial en las políticas públicas redistributivas guiadas desde una postura ética.
\end{abstract}

Palabras clave: Teoría de la Justicia, Modelos, Teoría de Juegos.

\section{Theory of Justice of Rawls. Amathematics-critic to Amartya Sen's critique of John Rawls}

\begin{abstract}
The article is framed on the analysis of Rawls's Theory of Justice from different perspectives. In this case, it refers to: (1) Amartya Sen's critique of some aspects of the model of Rawls; and (2) the response to these arguments from structuralist metatheory and the mathematics of games. We argue that Sen's position is weakened by changing the initial conditions of Rawls's model, and the lean mathematical results of the correction proposed by Sen. The result is that the model of Rawls is not bettered by the criticism of Sen and forces to consider it from an ideal normative perspective. In this perspective the math calculation is not discarded, which is essential on redistributive public policies guided from an ethical perspective.
\end{abstract}

Keywords: Theory of Justice, Models, Theory of Games. 


\section{Teoria da justiça de Rawls \\ Uma crítica-matemática à crítica de Amartya Sen a John Rawls}

Resumo: O artigo se insere na análise da Teoria da Justiça de Rawls a partir de diferentes perspectivas. Neste caso, refere-se a: (1) a crítica de Amartya Sen a alguns aspectos do modelo de Rawls; e (2) a resposta a estes argumentos desde a metateoria estruturalista e a matemática dos jogos. Argumenta-se que a posição de Sen é enfraquecida pela mudança das condições iniciais do modelo de Rawls, e pelos escassos resultados matemáticos da correção proposta por Sen. O resultado é que o modelo de Rawls não melhora pela crítica de Sen e força a considerá-lo a partir de uma perspectiva ideal normativa. Nesta perspectiva, não é descartado o matemática, o qual é essencial nas políticas públicas redistributivas guiadas a partir de uma postura ética.

Palavras-chave: Teoria da Justiça, Modelos, Teoria dos Jogos.

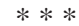

Lord Mansfield [...] dio un famoso consejo a un gobernador colonial recién nombrado: “Considere lo que usted crea que la justicia demanda y actúe en consecuencia. Pero nunca dé sus razones; pues su decisión será probablemente correcta, pero sus razones serán ciertamente erróneas”.

(Sen , 2010, pág. 36)

\section{Introducción}

El diseño de modelos teóricos, implica supuestos, axiomas y reglas de operación. El análisis de los modelos teóricos se centra en dos cuestiones: auto evidencia de los axiomas y consistencia del sistema. Si el modelo busca desprender de condiciones teóricas argumentaciones empíricas o conclusiones empíricas es necesaria una estrecha vinculación entre ambos. Aunque desprender conclusiones empíricas a partir de enunciaciones teóricas es sumamente cuestionable aunque contengan connotaciones empíricas (Balzer, Teorías empíricas: modelos estructuras y ejemplos, 1997) (Balzer, Moulines, \& Sneed, An Architectonic for Sciences: The Structuralist Program, 1987).

Un modelo teórico en Ciencias Sociales es relevante cada vez que este guarda sentido con la realidad a la que pretende explicar o dictar recomendaciones que bien pueden convertirse en pronósticos. Es decir, todo modelo teórico de la ciencia social es un modelo que implica un desarrollo como teoría empírica, puesto que se pretenden básicamente dos cosas: 1) generar explicaciones de la realidad y 2) generar pronósticos sobre dicha realidad, aunque no es lo que piensan autores destacados como Milton Friedman. ${ }^{1}$ Tampoco sucede así desde otras perspectivas trascendentales ${ }^{2}$ o ideales históricas ${ }^{3}$. 
Por ello, un modelo teórico que busque generar recomendaciones empíricas, como lo es el caso del modelo de Justicia de Rawls, tiene que obedecer a los lineamientos de generación de teorías empíricas, donde, a través de este pueda ser explicada la realidad y puedan generarse predicciones: tomando en consideración que las predicciones están limitadas por su dependencia del pasado (Rawls, 2006[1971]; Rawls, 1995; Rawls, 2006[1971]). Lo mismo podría suceder con las tesis abiertamente adversarias a Rawls (Nozick, 1988; Leriche \& Caloca, 2009).

Ello implica que el científico social buscará generar explicaciones antes que predicciones; a menos de que sea un instrumentalista que pretenda que dicha explicación redunde en una conexión no falsa entre la teoría y la realidad y cuya eficiencia sea sumamente alta, es decir que con la teoría se logre explicar la mayor parte de los fenómenos que caen dentro de su dominio.

Aceptando el supuesto anterior, sin necesariamente asumirlo, la presente investigación se elaboró con la intención de mostrar matemáticamente la inconsistencia o contradicción del esquema rawlsiano en sus supuestos y la matematización de la propuesta de Sen al respecto: que implica un distanciamiento de las condiciones iniciales del modelo de justicia de Rawls (Rawls, Teoría de la justicia, 2006[1971]).

Para lograr esto se plantean las siguientes cuatro secciones: en la primera se expone el planteamiento desde la teoría estructuralista de la ciencia, acerca de la división entre una parte conceptual y una de aplicaciones que contienen el conjunto de teorías científicas, y contemplando la existencia de modelos potenciales y potenciales parciales para la concurrencia entre teoría y observación. Posteriormente, en la segunda sección, se expone el dilema de los axiomas o de las condiciones iniciales de un modelo teórico con pretensiones empíricas dentro del esquema de las ciencias sociales.

Para en la tercera sección argumentar matemáticamente sobre la contradicción de las condiciones iniciales de la Teoría de la Justicia de Rawls, y cómo también en la crítica de Sen a Rawls --que presentamos a través de la teoría de juegos-- tales condiciones iniciales requieren de ajustarse. Finalmente exponemos nuestras razones acerca de cómo la crítica de Sen es una estratagema tradicional puesto que modifica el sentido de las condiciones iniciales para que sea adecuado el modelo de Rawls a lo que Sen establecería como lo "correcto" es decir hacer otro tipo de modelo que no sea esencialmente el de justicia de Rawls ${ }^{4}$.

\section{Ciencia empírica}

Para nuestros fines parte significativamente importante es la elucidación acerca de los núcleos teóricos en ciencia empírica, esto integra tanto a los objetos reales como a la idealización de los mimos para su estableci- 
miento teórico, es decir efectuar el complejo paso del lenguaje observacional al lenguaje teórico. Ahora, a continuación se presenta la arquitectónica de los núcleos teóricos constituidos por una idealización y sus aplicaciones que permiten el nexo entre lo teórico y el entorno empírico para ello nos basamos en Balzer (Balzer, Teorías empíricas: modelos estructuras y ejemplos, 1997; Balzer, Moulines, \& Sneed, An Architectonic for Sciences: The Structuralist Program, 1987).

En un primer momento y siguiendo a Diederich y Fulda (Diederich \& Fulda, 1981), se presentan las nociones básicas para la estipulación de un teoría empírica, esto a través del esquema estructuralista de las teorías de Balzer, Moulines y Sneed (Balzer, Moulines, \& Sneed, An Architectonic for Sciences: The Structuralist Program, 1987). Donde, una teoría en sí misma no es considerada como una aserción, ni tampoco una proposición sobre la que pueda ejecutarse un valor veritativo, sino que debe ser considerada como un par $<\mathrm{K}$, I $>$, cuyo primer componente $\mathrm{K}$ corresponde con la estructura conceptual de la teoría y el segundo componente I refiere al dominio de las aplicaciones propuestas para esa estructura.

Donde, el K de una teoría no determina su dominio de aplicaciones I, sino un dominio, normalmente no previamente dado, de "entidades" de las cuales la estructura conceptual $\mathrm{K}$ puede efectivamente predicarse dando proposiciones verdaderas, como lo es el dominio $\mathrm{M}$ de modelos de K. Aunado a ello, K determina el dominio de aquellas "entidades" sobre las cuales tiene sentido el preguntarse si K se aplica a ellas o no, es decir, el dominio $\mathrm{M}_{\mathrm{p}}$ de los modelos potenciales de $\mathrm{K}$, es decir, los modelos que pudiesen ser parte de $\mathrm{K}$.

Esto implica que para poder diferenciar estos dos dominios; el de los modelos efectivos de $\mathrm{K}$ y el de los modelos sobre los que posiblemente se pueda aplicar K, se requiere que K mismo este compuesto por dos partes, la primera que caracterice únicamente los modelos potenciales y la segunda que formule las leyes adicionales aplicadas a las "entidades" que caen dentro del dominio de los modelos.

Así, también es necesario reconocer que I no viene determinado lógicamente por $\mathrm{K}$ como en el caso de los $\mathrm{M} \mathrm{y} \mathrm{M}$, sino que se requiere que sea escogido adicionalmente bajo puntos de vista pragmáticos, pero dentro del marco fijado por K, esto como delimitación de un dominio de fenómenos a los que se busca aplicar K. Ello implica que los términos de I son más concretos que los contenidos en $\mathrm{K}$, puesto que derivan de la experiencia.

Ahora, para la teoría estipulada, le corresponde una aserción de la teoría que implica que los elementos de I puedan interpretarse como modelos M de K, ello, a partir de la construcción de una complemento conceptual adecuado para los elementos del dominio de I. Esta aserción ligada a la teoría no se divide en aserciones de aplicación aisladas para los 
elementos de I, sino que la teoría conecta entre sí estos elementos o aplicaciones a través de las condiciones de ligadura. Las condiciones de ligadura pudiesen para un mismo individuo, que aparece en múltiples aplicaciones, otorgarle valores numéricos funcionales iguales en cada una de las aplicaciones de la teoría. Es decir, esto permite conectar o ligar las aplicaciones a través de la teoría, ello implica que sea posible tener un cuerpo teórico sobre el que se ejecutan las aplicaciones y que no sea cualquier aplicación parte del cuerpo de corroboración de la teoría, lo cual incluye tanto a los modelos como a los posibles o potenciales modelos de dicho $\mathrm{K}$.

Por otra parte, en $\mathrm{K}$ aparecen, entre otros, los términos teóricos, cuyas características no necesariamente les excluyen del lenguaje observacional. Donde, si un concepto que aparece en K es teórico, no lo es en un sentido absoluto que trascienda a la T-teoría, sino sólo en relación a $\mathrm{T}$, es decir, es T-teórico. Claro es, que en K están contenidos conceptos que por ser teóricos, no pueden aplicarse o comprobarse directamente por los objetos del dominio de I o con base en las aplicaciones pragmáticas. Esto implica que no existe posibilidad de corroboración de los mismos, en cierto sentido se consideran por algunos miembros del empirismo lógico como metafísicos, ello es todo un debate en el que no existe espacio para aclarase aquí.

Esto es así, porque ni K ni la determinación pragmática de su I correspondiente nos permiten plantear significativamente, respecto de un objeto del dominio de I, si este es un modelo M de K o no. Sin embargo, la solución a esto se plantea a través de la propuesta del enunciado de Ramsey (Stegmüller, 1979), en donde, se estipula un enunciado cuya potencia deductiva no es menor, puesto que contiene la aplicación de $\mathrm{K}$ a tales objetos de I, es decir, es un enunciado que permite un puente "especialmente" conector entre K e I. Su derivación corresponde con el aislamiento de la estructura conceptual K' que resta cuando se aísla de los términos teóricos de $\mathrm{K}$, obteniéndose un nuevo dominio de "entidades”, el dominio $\mathrm{M}_{\mathrm{pp}}$ de los modelos potenciales parciales de K. Para los cuales puede argumentarse si pertenecen o no a un objeto del dominio de I. Es decir, permite la concatenación entre entidades conceptuales y su visión en la experiencia.

En este sentido, si la respuesta a la cuestión anterior es afirmativa, ergo, se asevera entonces que cada $\mathrm{M}_{\mathrm{pp}}$ de $\mathrm{K}$ que pertenezca a I puede completarse teóricamente hasta convertirse en un modelo de $\mathrm{K}$, es decir, existe un $\mathrm{M}_{\mathrm{p}}$ cuya parte no-teórica es un $\mathrm{M}_{\mathrm{pp}} \mathrm{y}$ tal que es un $\mathrm{M}$ de $\mathrm{K}$ (véase diagrama 1$)^{\mathrm{p}}$ 


\section{Diagrama 1: Modelos y Modelos potenciales}

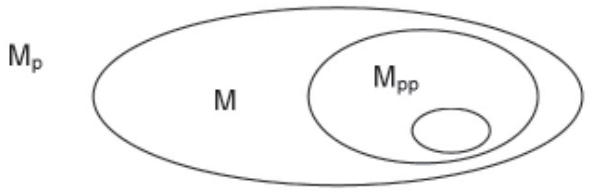

Fuente: Adecuación propia con base en (Balzer, Teorías empíricas: modelos estructuras y ejemplos, 1997)

Ahora bien, parte importante para la disertación tiene que ver con la estipulación de que una teoría T concebida como el par $\langle\mathrm{K}$,I $>$ que en su despliegue o extensión se da en dos niveles; por una parte, la teoría se desarrolla a nivel teórico por ampliación y diferenciación de la estructura conceptual de K y por otra parte, a nivel empírico se desarrolla por extensión del dominio de aplicación I. Es decir, una teoría se desarrolla ya sea por contemplar un mayor campo de conceptos y por refinamiento de algunos y/ o por extender su campo de aplicación a nuevos esquemas empíricos que puedan abordarse con ella.

La ampliación teórica de K se efectúa al añadir a la estructura conceptual ya existente nuevas leyes especiales en forma de enunciados abiertos, así como condiciones de ligadura especiales. La extensión del dominio de aplicación I da el recorrido de las variables que aparecen en K una interpretación por medio de conceptos no-teóricos, manteniendo el puente antes dicho.

En este sentido, si se tiene $E_{1}, E_{2}, \ldots$ una sucesión de ampliaciones de $\mathrm{K} \mathrm{e}_{1}, \mathrm{I}_{2}, \ldots$ una sucesión de ampliaciones de I; entonces, la doble evolución de la teoría puede simbolizarse mediante las cadenas:

\section{$\mathrm{E}_{0} \leq \mathrm{E}_{1} \leq \mathrm{E}_{2} \leq \mathrm{E}_{3} \leq \ldots \mathrm{E}_{\mathrm{j}} \leq \ldots$ y $\mathrm{I}_{0} \subseteq \mathrm{I}_{1} \subseteq \mathrm{I}_{2} \subseteq \mathrm{I}_{3} \subseteq \ldots \mathrm{I}_{\mathrm{k}} \subseteq \ldots$}

Donde, cada E y cada Ik son ampliaciones de los anteriores, así, a cada $E_{j}$ le corresponde un conjunto $\mathrm{A}\left(\mathrm{E}_{\mathrm{j}}\right)$ de dominios en los que la aplicación dẻ K es realmente válida.

Sí el núcleo $\mathrm{E}_{0}$ y el dominio de aplicación $\mathrm{I}_{0}$ se amplían hasta los puntos $\mathrm{E}_{\mathrm{j}}$ e $\mathrm{I}_{\mathrm{b}}$, entonces la aserción empírica de la teoría viene dada por: $\mathrm{I}_{\mathrm{k}} \bullet \bullet$ $\mathrm{A}\left(\mathrm{E}_{\mathrm{j}}\right)$, de tal suerte que al ir aumentando $I_{k}$, se afirma una aplicabilidad mayor, mientras que al irse diferenciando Ej a nivel teórico se hacen aseveraciones cada vez más específicas acerca de las aplicaciones propuestas, ello implica una refinación de la teoría. Puede suceder que ante una ampliación teórica esta vaya asociada a una aplicación empírica. Es decir, que cada vez que se amplía la teoría también se amplíe el espectro de aplicación, con ello nuestro cuerpo teórico $<\mathrm{K}$,I $>$ se expande y puede abarcar un número mayor de refinamientos con la extensión del campo de explicación. 
Por otro lado, es necesario comentar que en nuestro caso la teoría referida a la justicia permite establecer una conexión entre su edificación teórica y sus relaciones empíricas bajo ciertas condiciones específicas, sin embargo, un punto a favor de Sen es que en el esquema rawlsiano el paso de la teoría a la experiencia es sumamente burdo: esto lo aclararemos en secciones más abajo.

\section{Qué es un modelo y la crítica a un modelo teórico sobre sus supuestos}

Ahora como preludio a la crítica matemática de la crítica de Sen a Rawls es necesario establecer las condiciones que nos conducen a estipular que las referencias de Sen al modelo de Rawls son pertinentes o no o bajo quë circunstancias lo son (Sen , La idea de la justicia, 2010).

Para ello es necesario aclarar en un principio qué consideramos como modelo y qué significa, en sus alcances, elaborar una crítica a un modelo con base en los supuestos o condiciones iniciales que le sustentan: claro es que también es necesario aclarar que los supuestos pudiesen no necesariamente jugar un papel de axiomas o de verdades autoevidentes.

Un modelo estrictamente es una abstracción de la realidad, es un conglomerado argumentativo lógicamente consistente que representa una idea de lo que es la realidad, ello es así porque no existe forma, hasta ahora, de hacer funcional una explicación sobre la realidad basada en el conjunto de hechos y la relación entre estos hechos.

Lo único a lo que los seres humanos tenemos acceso, son a referentes que guían nuestras interpretaciones explicativas acerca de lo que es el mundo. Por ende, nuestro acceso a los hechos sociales o en su caso a los hechos de la experiencia que atiende la justicia es a través de una idealización de lo que es la teoría de la justicia.

En este sentido, la teoría de la justicia es un modelo ideal, abstracción de las condiciones de la realidad que sirve para explicar a la misma y generar en segundo término predicciones sobre esta. Sin embargo, a un bajo estas condiciones surge un aspecto esencial sobre la teoría de la justicia: ¿ esta debe contener axiomas o supuestos o condiciones iniciales para ser operativa?

En primer término exploramos la idea de que los supuestos de una teoría social como lo es la teoría de la justicia puedan llamarse axiomas. En realidad rechazamos la probabilidad de que esto ocurra, sencillamente porque los axiomas están sustentados bajo la noción de que son verdades autoevidentes, y en ciencias sociales mucho de lo que se hace con modelos teóricos emitidos bajo un lenguaje natural y no así artificial como el de las matemáticas o la lógica corresponde con argumentaciones con contenido 
significativo, es decir muy bien pueden ser argumentaciones verdaderas o falsas que funcionan para erigir el edificio o constructo teórico, siendo pretensiones explicativas o predictivas: como en el caso del instrumentalismo donde los supuestos pueden ser falsos siempre y cuando las predicciones sean acertadas.

De igual manera, basta recordar que en ciencias sociales algunas teorías en su totalidad están conformadas por condiciones básicas que son falsas, lo cual rechaza la probabilidad de que todo supuesto en ciencias sociales pueda ser admitido como un axioma de la teoría. Lo que es factible es establecer que los supuestos de una teoría puedan considerarse como las condiciones iniciales para el funcionamiento y construcción de una teoría: son los cimientos.

Por ende, es que se requiere volver los ojos a las cuestiones de si consideramos o no como factible la probabilidad de que las condiciones iniciales de un modelo teórico sean verdaderas o puedan ser falsas. De primera instancia nos preguntaríamos acerca de la deducibilidad de argumentos verdaderos a partir de condiciones iniciales falsas. Esto no visto estrictamente como proposiciones lógicas sino como argumentos que en muchos de los casos lo que forman son explicaciones causales, esto es, ¿podemos generar explicaciones causales verdaderas a partir de condiciones iniciales falsas?

En realidad existe la probabilidad de que esto sea así, pues no sea demostrado de que en todo caso de teoría social con condiciones iniciales o supuestos falsos no se hayan podido generar explicaciones certeras. Sin embargo, el problema que surge aquí es cuando nos preguntamos acerca de la teoría científica en ciencia social ¿por qué? Simplemente, porque como a través de supuestos falsos se tienen que formar argumentaciones teoréticas que pueden ser corroboradas por la realidad es decir, el supuesto puede ser falso pero el edificio estructural de la teoría tiene que estar conformado por formaciones que encuentren un correlato con la experiencia sino la teoría dejara de corroborarse y por ende, la teoría será falsa y lo que tendríamos es que teorías formadas a partir de supuestos falsos en algunos casos terminan por ser falsas.

Por supuesto que el instrumentalismo es un caso en el cual aún con supuestos falsos es probable tener predicciones corroborables por la realidad. Empero, bien sabemos que lo más importante de una teoría cuando hacemos abstracción de la realidad es la de generar explicaciones sobre esa realidad no el que las teoría nos sirvan como medios para el fin último de la predicción, pues bien el futuro es incierto y en extremo cambiante pues los seres humanos somos criaturas intencionales.

En todo caso la predicción tendría que ser lo más certera posible. Pero qué sabemos acerca de las predicciones: que estas no tienen un alto grado de precisión y que si llega a ellas mucho obedece a la obra de las posibilidades infinitas de ocurrencia casual de un evento y por ende, de la 
puntería que se tenga para el logro de lo estipulado y no a una obediencia ciega de las leyes de la ciencia, ello por supuesto para las Ciencias Sociales, aunque para las Ciencias de la Naturaleza opera el principio de incertidumbre de Heisenberg, y en la lógica-matemática el teorema de incompletitud de Gödel $^{5}$.

Por qué el futuro es casual y no causal en cierto sentido. Esto se debe a que para formarnos una idea de cómo será el futuro nos basamos en los hechos pasados y presentes, es decir, nos basamos en la información incompleta que esta a nuestra disposición, y el futuro es una entidad con información incognoscible por los humanos, porque no está a nuestra disposición.

Si por ejemplo el pasado esta descrito por $\mathrm{T}_{\mathrm{t}-1}$, el presente por $\mathrm{T}_{\mathrm{t}} \mathrm{y}$ el futuro por $\mathrm{T}_{\mathrm{t}+1}$, tenemos que en una visión determinista:
1) $T_{t+1}=T_{t}+T_{t-1}$,
2) $T_{t}=T_{t+1}-T_{t-1} y$
3) $T_{t-1}=T_{t+1}-T_{t}$

Empero si el futuro no ha ocurrido como saber a que restar en 2) del pasado y en 3) a que restar el presente, necesitamos conocer el futuro para realizar dichas operaciones. Sólo en una visión determinista sabríamos que implica necesariamente el que el futuro sea efectivamente la suma del pasado y del presente. Sin embargo, por definición el futuro es desconocido, por ejemplo en una visión que toma en cuenta la incertidumbre del futuro nuestra condición es la siguiente:

Si por ejemplo el pasado esta descrito por Tt-1, el presente por Tt y el futuro por $\mathrm{Tt}+1+\mathrm{f}$, tenemos que en una visión indeterminista $(\phi)$ :

1) $\mathrm{Tt}+1+\phi \neq \mathrm{Tt}+\mathrm{Tt}-1$,

2) $\mathrm{Tt} \neq \mathrm{Tt}+1+\phi-\mathrm{Tt}-1 \mathrm{y}$

3) $\mathrm{Tt}-1 \neq \mathrm{Tt}+1+\phi-\mathrm{Tt}$

Esto es así simple y sencillamente porque desconocemos cuál es el contenido informacional de f y con ello, se puede claramente establecer que con el simple hecho de que reconozcamos aunque sea una ínfima parte de incertidumbre en el presente sobre lo que ocurrirá en el futuro ergo no podremos determinar el futuro única y exclusivamente a través del pasado y el presente.

Lo anterior nos lleva a la infactibilidad de que consideremos a nuestras teorías como simples medios o instrumentos para la predicción. Esto implica que considerar condiciones iniciales falsas bajo la idea de que lo significativo son las predicciones es ineficiente en cualquier sentido. Porque la predicción no es fructífera. 
Es decir, es infactible para la explicación y para la predicción considerar condiciones iniciales falsas pues conducen a decir: que da lo mismo utilizar esas condiciones iniciales o ninguna, y esto es de mayor relevancia si aún a pesar de la ineficiencia del procedimiento incurrimos en decir que es factible deducir a partir de argumentos teóricos falsos explicaciones empíricas verdaderas. En realidad, de hacerse esto, lo que ocurre es un "pase mágico”, que no es parte del proceso científico.

Por otra parte, existen mecanismos de crítica de un modelo con base en sus supuestos que enuncian que un modelo es limitado en su cobertura de explicación sólo por el hecho de que tiene unas condiciones iniciales en vez de otras. Nuestra opinión al respecto es que esto es una crítica sumamente débil, debido a que yo puedo plantear tres modelos el modelo con poder explicativo A con unas condiciones iniciales 1 (A1) un modelo B con iguales condiciones iniciales 1 y menor poder explicativo (B1) y un modelo $\mathrm{C}$ con condiciones iniciales diferentes 2 y un poder explicativo mayor que $\mathrm{A}$ ó B (C2) y argumentar que a mi parecer si cambio las condiciones iniciales de B por 2 (B2) entonces su poder explicativo será mayor al de A1. En realidad esto no puede ser una mera opinión sino que tiene que sustentarse en la corroboración de que efectivamente al cambiar las condiciones iniciales de B1 a B2 este explica más que A1.

El dilema aquí es que si esto ocurre de esta manera, entonces B1 obviamente ya no es B1 sino que su poder explicativo se transformo, entonces llegará a ser D2, es decir, otro modelo totalmente diferente. De ser esto así entonces porque argumentar que un modelo debe -normativamente-, cambiar sus supuestos si es posible plantear un nuevo modelo D2 sin recurrir al caso de transformación de B1. Una vez explorado lo anterior se requiere estipular la principal argumentación de rechazo por parte de Sen al modelo de Rawls sobre la teorçia de la justicia.

\section{La argumentación de Sen respecto a las condiciones iniciales del modelo de justicia de Rawls}

La crítica de Sen al modelo de la teoría de la justicia contractualista de Rawls, corresponde principalmente con un ataque a las condiciones iniciales con las cuales opera la construcción del modelo rawlsiano. Para Sen, Rawls elabora un planteamiento inadecuado sobre la teoría de la justicia al remitirse a solucionar dilemas con base en las instituciones antes que fijarse el objetivo central de toda teoría de la justicia que son los individuos.

Sin embargo, el planteamiento de Sen tiene larga data en sus escritos, porque de nueva cuenta condiciona su explicación a las llamadas capacidades, es decir facultades que posee cada individuo que distan del planteamiento rawlsiano contractualista: de velar por uno en el contexto de los demás, que permiten que uno pueda salir de una situación adversa y supuestas en dos momentos: primero es necesario generar capacidades en las 
personas para que en un segundo término se generen las condiciones apropiadas para que las personas que han adquirido capacidades puedan hacer uso de estas. Son dos formas distintas de ver los parámetros de cooperación, es decir, en Rawls cuando formo parte del contrato estoy cooperando con los demás en el mismo momento en que estoy actuando a mi favor y en el de Sen ocurre no instantáneamente sino que obtengo capacidades pensando en mi y luego coopero con los demás, aunque para ello requiera que los demás piensen en mi pero antes pensaron en ellos mismos.

Este obtener capacidades en el esquema seniano tiene una connotación social puesto que si estas posteriormente no se pueden usar por un impedimento del mercado o del Gobierno para generara las condiciones que puedan favorecer el uso de las mismas entonces, se señala como una hecho de ineficiencia en el camino trazado para sustraer a las personas de condiciones desfavorables como la pobreza en sentido amplio. Es decir, de una pobreza tanto absoluta como relativa.

La generación y uso de dichas capacidades implicaría la posibilidad de que las personas también puedan no sucumbir de los efectos adversos que contienen situaciones de pobreza como es la injusticia. Esto porque el hacerse de capacidades y uso de ellas va acompañado necesariamente de tres factores: la elevación de su nivel educativo de las personas, aumento en el nivel de ingreso y el empoderamiento tanto de hombres como de mujeres, a los hombres respecto de su exterior y a las mujeres tanto en su hogar como en su trabajo extradoméstico.

Sen está pensando en sus contribuciones acerca de que la generación y uso de capacidades necesariamente ve acompañado del lograr alcanzar una serie de funcionamientos que pueden estar rigiendo la probabilidad de realización de la persona en particular. Donde, los funcionamientos no son otra cosa que condiciones tanto materiales como vinculadas a los intereses de la persona, es decir, abarcan tanto la adquisición de alimentos básicos como la elevación de la autoestima o alcanzar la visión particular de felicidad, con el objetivo de que una vida sea digna de ser vivida.

Al establecer Sen esto está fijando la vista en su particular modelo de apreciación abstracta de la realidad, basada en supuestos no falsos hasta ahora. Por ello, se concentra en los resultados de la teoría de la justicia de Rawls y en las condiciones iniciales -sobre las cuales hablaremos más abajo. En este sentido, el esquema rawlsiano se interesa por las instituciones formadas por grupos de individuos y el seniano por individuos en particular.

En el caso de su crítica referente a que el modelo de Rawls se aboca en mayor medida a las instituciones aunque toma ciertos elementos que hacen referencia a los individuos, Sen esta convencido de que es el camino equivocado pues si bien se tiene que tener referencia a las instituciones en mayor medida se debe elaborar un planteamiento de la teoría de la justicia relacionado con las personas. 
En este sentido, proponemos un modelo matemático basándonos en la teoría de juegos para la presentación de la mayor relevancia de cada una de las posturas: Sen-Rawls así como la posibilidad de encontrar una solución equilibrada ante tales circunstancias. $\mathrm{O}$ al menos de mostrar cuál es la probabilidad de que cada enfoque presente la mayor parte de las veces su postura.

El juego se delimita de la siguiente manera: El juego es un tipo Guerra de los Sexos pues en este planteamiento se permiten obtener soluciones probabilísticas para las estrategias aunado a que se toma en consideración que ambos teóricos van de la mano en la explicación particular sobre lo qué es la teoría de la justicia. Los jugadores son nuestros dos teóricos: Amartya Sen y John Rawls, cada uno tiene dos estrategias que son personas e instituciones y los pagos corresponden con un mayor nivel para aquella opción que han preferido principalmente para sus explicaciones: personas Sen (5), Instituciones Rawls (5), y el valor menor a este y distinto de cero (1) para aquella opción que no es la principal para cada una de las estrategias de estos: instituciones para Sen (1) y personas para Rawls (1). Ahora como necesariamente indicamos que ambas propuestas ocurren en un momento histórico contemporáneo en el que pueden ser debatidas de manera competitiva entonces en aquellas opciones que se ubican donde ni Sen ni Rawls optarían son: Instituciones Sen y personas Rawls $(0,0)$ primera columna segunda fila, así mismo, en donde cada uno se quedaría únicamente con una parte de la explicación lo cual no ocurre porque si bien ambos optan preferentemente por una opción no se alejan de la otra, esto sería con personas Sen e instituciones Rawls $(0,0)$ segunda columna primera fila (véase matriz 1) (Rawls, Teoría de la justicia, 2006[1971]) (Sen , La idea de la justicia, 2010).

MATRIZ 1

RAWLS

\begin{tabular}{|c|c|c|c|}
\hline \multirow{3}{*}{ SEN } & & PERSONAS & INSTITUCIONES \\
\hline & PERSONAS & $(5, \underline{1})$ & $(0,0)$ \\
\hline & INSTITUCIONES & $(0,0)$ & $(1, \underline{5})$ \\
\hline
\end{tabular}

Fuente: Elaboración propia.

Aquí nos encontramos con dos subequilibrios de Nash en estrategias puras, los cuales corresponden con los mayores valores fila y columna subrayados $(5,1)$ y $(1,5)$, sin embargo se debe de contar con la unicidad del equilibrio de Nash. Para ello, se emplea otro método que nos permite encontrar el equilibrio único de Nash y esto es a través de estrategias mixtas (véase matriz 2). En términos de la situación experimentada lo que se observa es que tanto o con qué frecuencia Sen opta por personas o por instituciones en su propuesta y Rawls que tanto opta por instituciones o perso- 
nas en su propuesta. Lo esperado es que los mayores valores probabilísticos para Sen estén en optar por personas y para Rawls que estén en instituciones sin dejar de largo que exista una probabilidad menor para la propuesta contraria en ambos casos. En este caso la X y 1-X serán las probabilidades con las que incurre Sen en una u otra categoría, para Rawls sus prolijidades estarán dadas por Y y 1-Y.

MATRIZ 2

RAWLS

\begin{tabular}{|c|c|c|c|c|}
\hline & & & & \multirow{3}{*}{$\begin{array}{c}\text { INSTITUCIONES } \\
1-\mathrm{y}\end{array}$} \\
\hline & \multirow{2}{*}{$\begin{array}{c}\text { PERSONAS } \\
\mathrm{Y}\end{array}$} & \\
\hline & & & & \\
\hline \multirow[t]{2}{*}{ SEN } & PERSONAS & $\mathrm{x}$ & $(5,1)$ & $(0,0)$ \\
\hline & INSTITUCIONES & $1-x$ & $(0,0)$ & $(1,5)$ \\
\hline
\end{tabular}

Fuente: Elaboración propia.

Una vez organizada la matriz de forma estratégica con las variables probabilísticas, procedemos a realizar los cálculos obteniendo el siguiente resultado (véase matriz 3).

MATRIZ 3

RAWLS

\begin{tabular}{|c|c|c|c|}
\cline { 3 - 4 } \multicolumn{2}{c|}{ SEN } & PERSONAS & INSTITUCIONES \\
\cline { 3 - 4 } \multicolumn{2}{|c|}{} & $\mathrm{y}=\frac{1}{6}$ & $1-\mathrm{y}=\frac{5}{6}$ \\
\hline PERSONAS & $\mathrm{x}=\frac{5}{6}$ & $(5,1)$ & $(0,0)$ \\
\hline INSTITUCIONES & $1-\mathrm{x}=\frac{1}{6}$ & $(0,0)$ & $(1,5)$ \\
\hline
\end{tabular}

Fuente: Elaboración propia.

Este resultado sustituidos por valores que pertenecen a los reales nos permiten la configuración del equilibrio único de Nash, lo que a continuación procede es mostrar como bajo estas condiciones es posible establecer en un plano el equilibrio de Nash y con ellos las probabilidades con las cuales optan por una estrategia antes que otra (véase gráfica 1). 


\section{Gráfica 1}

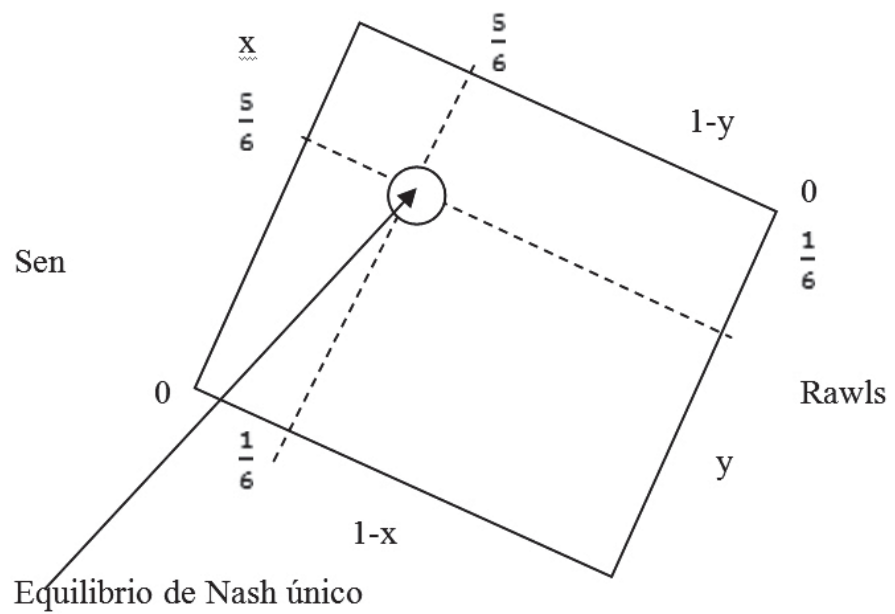

Fuente: elaboración propia.

Como puede observarse Sen opta por su estrategia explicación de la teoría de la justicia basado principalmente en las personas con una probabilidad de $\frac{5}{6}$, y por su estrategia explicaciones sobre la teoría de la justicia con base en instituciones con $\frac{1}{6}$, en el caso de Rawls lo esperado era todo lo contrario sus explicaciones sobre teoría de la justicia con base en personas son elegidas con una probabilidad de $\frac{1}{6}$, y con base en instituciones con una probabilidad de $\frac{5}{6}$.

En este caso el equilibrio puede asignarse al encontrar las ecuaciones generales de equilibrio que nos indican que para diferentes valores de x,y pueden configurarse las siguientes trayectorias de [0,1], y con ello mostrar cómo evolucionan las probabilidades de las propuestas hasta encontrar un equilibrio entre las dos estrategias por las que optan en sus explicaciones sobre la teoría de la justicia tanto Sen como Rawls (véase gráfica 2).

Donde las ecuaciones corresponden con:

$$
\begin{aligned}
& Z_{1}=6 x y-(x+y)+1 \\
& Z_{2}=6 x y-5(x+y)+5
\end{aligned}
$$




\section{Gráfica 2}

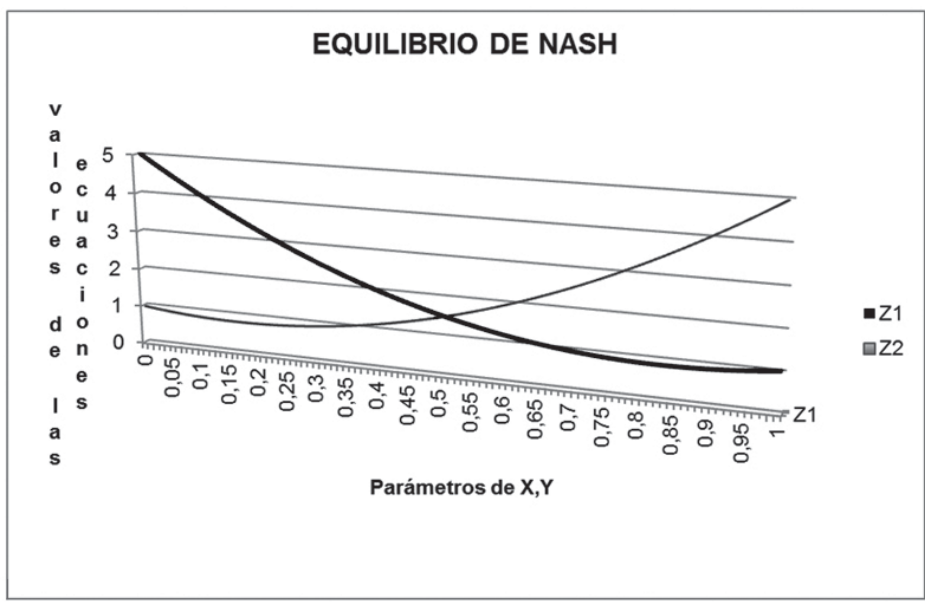

Fuente: elaboración propia.

En este sentido, las argumentaciones de Sen y Rawls podrían bien balancearse para formar una tercera teoría de la justicia que responda también al equilibrio único. Por el momento lo que queda en claro es que Sen recurre a su explicación basándose en las personas el 83.33 por ciento de las veces y Rawls recurre a su explicación basándose en instituciones el 83.33 por ciento de las veces.

Esto es fundamental para establecer que el planteamiento seniano es que los individuos son primero, pero esto depende del tipo de perspectiva adoptada, que en el caso de Sen deviene de su apego al individualismo metodológico.

\section{Los argumentos críticos respecto de la crítica de Sen al esquema de Rawls}

Ahora es necesario mostrar los planteamientos críticos a la crítica de Sen. En este caso recurriremos principalmente a lo que identificamos como el dilema de Sen basándonos en la sección matemática elaborada más arriba que trata sobre la crítica de un modelo con base en sus supuestos y la transformación de un modelo a otro totalmente distinto, así como la determinación de la estructura de una teoría científica.

Sen elabora una crítica al modelo de Rawls básicamente orientada a la consideración de unos resultados guiados por sus condiciones iniciales, es decir, se afirma que Rawls obtiene dichos resultados orientados a institu- 
ciones principalmente remitiendo de manera marginal a las personas porque escoge unas condiciones iniciales de un tipo determinado que, añadimos nosotros, son falsas (Sic.).

Sin embargo Rawls se ha defendido al respecto aludiendo que sus condiciones iniciales o posición original podrían bien ser desechados y seguir vigentes los principios de la justicia obtenidos. Pero con base en la argumentación de Barry (Barry ,. , 1997; Barry B. , La teoría liberal de la justicia, 1993; Barry B. , Teorías de la justicia, 1995[1989]) podemos rechazar la idea de la posición original y veremos que sin esta la probabilidad de obtener los principios de la justicia es de sólo un 25\% lo cual nos indica que con una probabilidad del 75\% se requiere de la posición original (falsa) para poder obtener los principios de la justicia y que con un $25 \%$ de la probabilidad, con certeza, podríamos obtener otros principios de la justicia y no los obtenidos por Rawls.

Puesto que si rechazamos la idea básica de la posición original existen dos posibilidades que aceptemos o que rechacemos los dos principios sobre la justicia (véase gráfica 3) en cuyo caso su rechazo tiene una probabilidad de ocurrencia de $1 \frac{1}{4}$ o del $25 \%$ lo cual es sumamente bajo. Es decir, la dependencia de que se elija la posición original es muy alta, por ende, cualquier crítica a la posición original (falsa) es sumamente efectiva para rechazar la teoría de la justicia de Rawls.

\section{Gráfica 3}

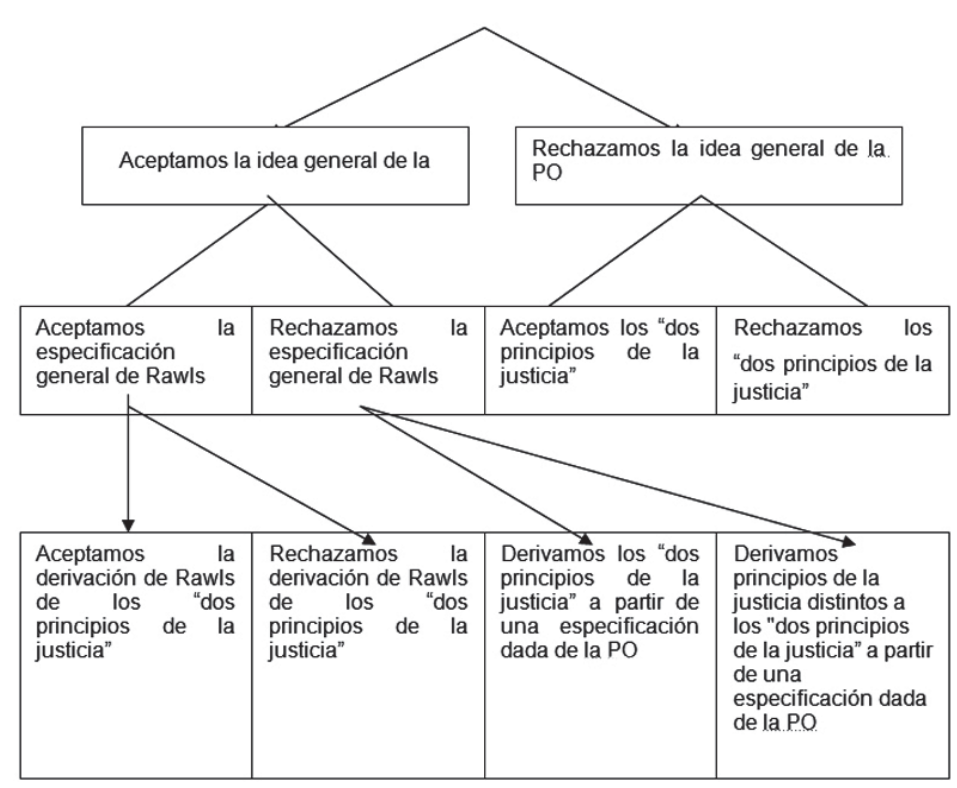

Fuente: Elaboración propia con base en (Barry; 1993: 60). 
Sin embargo, Sen opta por el camino más simple en la elaboración de una crítica: "yo creo que para que este enfoque sea correcto se debe optar por el tipo de explicación que yo brindo”. Es un enfoque del todo lo que no se ajuste a mi explicación esta incorrecto. Es por ello que lo hemos llamado la "crítica débil" pues su argumentación redunda en que para transformar dichas condiciones de Rawls para enfocarse en las personas las condiciones iniciales del modelo de Rawls deben de tomar en cuenta, normativamente: es decir por la crítica subjetiva de Sen, la noción de capacidades.

Esto conlleva a una sola solución; para que el modelo de Rawls sea operativo debe transformarse en otro modelo ¿qué entonces no podría de origen plantearse mejor otro modelo? Y de ser así, según Sen, debería ser el suyo: referente a condiciones iniciales que optan por obtener capacidades para alcanzar funcionamientos y con un enfoque orientado a las personas. Pero este es el modelo de Sen, entonces para que el modelo de Rawls sea compatible con la crítica de Sen tiene que transformarse en el modelo del tipo de Sen; esto sin lugar a dudas es transformar el modelo B1 en un D2 que es totalmente diferente. Por lo tanto la crítica de Sen es sumamente débil: para que un modelo sobre la justicia sea acertado debe de ser de entrada del tipo D2 es decir, como el que yo Sen planteo. Así, para Sen cualesquiera modelos de justicia tendrán de entrada que ser del tipo D2.

Lo que es de mayor factibilidad es hacer una crítica de las condiciones iniciales del modelo de Rawls, relacionado con lo falso de sus supuestos ya que un modelo así sería instrumental, y lo que interesa en este tipo de modelos no son las explicaciones sobre las condiciones de la teoría de la justicia sino lo que podemos predecir en un futuro que podrían ser las condiciones o principios de la justicia: los dos principios de la justicia, pero ello remite a dos cuestiones: 1) el modelo de Rawls atiende al futuro y 2) las condiciones iniciales del modelo son falsas y hasta contradictorias.

En el primer caso se tiene que de atender al futuro, basándonos en el pasado únicamente, implica que no existe forma de integrar la mínima proporción de incertidumbre, que al incorporarse implicaría un resquebrajamiento del modelo de Rawls, porque con incertidumbre por parte de los individuos de la posición original; estos no necesariamente podría elegir los principios de la justicia que deriva Rawls. Esto porque tendría que tomar en consideración la posibilidad empírica de que el contexto influye para que no se den dichos principios. Esto redunda en 2).

Ahora, el modelo teórico de Rawls, es un modelo que corresponde al tipo de modelos que trabajan con condiciones iniciales o supuestos falsos, esto redunda que se esperaría de este más una predicción certera que una explicación sobre la justicia, esta explicación certera corresponde básicamente con la derivación de los principios de la justicia. Pero dicho principios de la justicia como derivados tienen dos posibles connotaciones: 1) son teóricos o 2) son empíricos.

En el primer caso la derivación es meramente teorética y por ende, 
ideal y sólo es una construcción abstracta que no tiene nada que ofrecer en el mundo las explicaciones para las teorías empíricas, pero si ocurre 2) entonces, tenemos que la predicción de nuestra teoría de supuestos falsos debiera ser certera. Empero veamos que enuncian los dos principios de la justicia: El primer principio de la justicia establece que cada "persona ha de tener un derecho igual al esquema más extenso de libertades básicas que sea compatible con un esquema semejante de libertades para los demás.” Y el segundo principio considera que las “desigualdades económicas y sociales han de ser estructuradas de manera que sean para: a) mayor beneficio de los menos aventajados, de acuerdo con un principio de ahorro justo, y b) unido a que los cargos y las funciones sean asequibles a todos, bajo condiciones de justa igualdad de oportunidades”.

De esto lo que sabemos es que las personas pueden tener derecho a cualquier cosa que no afecte a la comunidad pero en la práctica no permitírseles ejercer este derecho a todos por igual como se enuncia en el primer principio y con referencia al segundo claramente conocemos que en el mudo no existe una justa igualdad de oportunidades. Esto lleva a que la teoría de la justicia de Rawls a través de su predicción de los principios de la justicia aceptando sus supuestos falsos no tiene referente empírico que la corrobore por el contrario hay un referente empírico claro que la hace falsa: La teoría de la justicia de Rawls como teoría social empírica es falsa.

Esto es, el planteamiento de Rawls es de tal estructura que el vínculo entre modelos potenciales parciales Mpp que puedan tener conexión con la parte conceptual (K), es decir, que la parte empírica o de las aplicaciones (I) no se conecta directamente de las explicaciones conceptuales pues estas son falsas en principio. Ello aunado a que en ciencia empírica los principios de la justicia deberían ser aplicables a la realidad de cualquier sociedad. Empero, el contexto claramente determina que tales principios no son del todo operativos en la realidad, lo cual sucede porque lo que se tiene con la propuesta de Rawls no es una explicación sobre la justicia sino una predicción ¿para qué sociedad? Para una ideal, por ende, la propuesta rawlsiana no deja el ámbito de la parte conceptual (K) de los modelos (M) para alcanzar la parte de las aplicaciones (I) sino que permanece conceptualmente ideal. La teoría de la justicia de Rawls no es una teoría empírica y si lo pretendía: es falsa.

Lo anterior sin dejar de pensar en que los supuestos de la teoría de la justicia de Rawls son falsos; en la sociedad no se parte de situaciones justas en principio, nuestras sociedades en su mayoría incurren en condiciones de injusticia. Y los individuos no son independientes de su identidad cuando analizan cualquier condición que les pudiese afectar. Además, los individuos no son sólo competitivos racionales y autointeresados como en la teoría económica, también son cooperativos y empáticos.

Todo ello aunado a que de la interacción social con individuos perfectamente racionales no siempre se obtienen resultados satisfactorios como lo indica el dilema del prisionero en teoría de juegos (véase Leriche y Caloca, 2009). 
Esto por supuesto nos indica los límites de la elección racional y con ello la no plausibilidad de que socialmente puedan elegirse principios de la justicia eficientes por el sólo hecho de contar con personas racionales en el sentido de la teoría económica.

Con todo lo anterior mostramos que las condiciones iniciales o la posición original de Rawls puede muy bien no resultar en lo esperado por él: es falsa y contradictoria, y que si bien existen limites a su explicación la solución no es del todo cambiar por completo las condiciones iniciales, puesto que si esto fuese el caso Sen tendría razón y su modelo de teoría de la justicia sería no objetivamente pero, si intersubjetivamente el aceptado por la comunidad experta.

\section{Conclusiones}

En primer lugar, se ha establecido a los modelos como una de las condiciones relevantes de la ciencia empírica para confrontar lo teórico conceptual y las aplicaciones de las teorías. En segundo se definió el carácter de los modelos y su crítica por sus supuestos. En tercero, se recurre al ejemplo de la crítica de Sen a Rawls para ilustrar estas cuestiones.

En el caso específico del artículo, la crítica de Sen a la teoría de Rawls releva que -"las condiciones iníciales”, "posición original”, o "principios de la justicia” hasta los resultados-son ideales y, que, por ende, la teoría no tiene un legítimo correlato empírico. La teoría de Sen a Rawls muestra que sus supuestos serían falsos, aunque a Rawls, no pareciera interesarle las explicaciones cuantitativas, contextuales universales sino afirmar a la justicia como un principio (o fin).

El cuestionamiento de Sen a Rawls es una "crítica débil” a los supuestos y resultados de la teoría de la justicia de Rawls. Básicamente, porque sólo indica que la teoría de Rawls estaría mejor si siguiera los supuestos de la teoría de Sen: la consideración de "las capacidades" orientados principalmente a las personas. Esta es una crítica débil puesto que para mejorar el modelo de Rawls se propone fabricar otro modelo pero éste sirve para refutar la no corroboración de la teoría original de Rawls. La "confianza” de Rawls en la "racionalidad" y en "individuos autointeresados", como se utiliza en la economía, se confabula para logros catastróficos de modo que sus principios de la justicia no sean corroborables experimentalmente y, por lo tanto, su teoría de la justicia como teoría empírica sea falsa.

Por último, Amartya Sen y John Rawls son cómplices en la recuperación de la idea de justicia como equidad en el cuadro del ilustrado filósofo y gran matemático Marie-Jean-Antoine Nicolas de Caritat, marqués de Condorcet. El ensayo de crítica de Sen busca la mejoría del modelo de Rawls, sin poder renunciar a una postura suave del rational choice. Pero, siendo un avance significativo la doctrina de las capacidades no lo es menos el trascentalismo kantiano de Rawls. Es lo que quiso mostrar el artículo en una antítesis que permanece contemporánea. 
Polis, Revista Latinoamericana, Volumen 14, $N^{\circ}$ 40, 2015

\section{Notas}

${ }^{1}$ Aunque "El avance de la economía positiva requerirá no sólo (...) si no de hipótesis nuevas. (...). La construcción de hipótesis es un acto creativo de inspiración, intuitivo, invención; su esencia es la visión de algo nuevo en el material conocido. El proceso deberá explorarse en categorías psicológicas, no lógicas; estudiarse en autobiografías y biografías, no en tratados sobre el método científico, y promoverse por la máxima y el ejemplo, no por el silogismo o el teorema" (Friedman, 1986, pág. 76)

${ }^{2}$ La importancia de la postura trascendental tiene su origen filosófico en Kant (Kant, Crítica de la razón práctica, 1999; Kant, Crítica de la razón pura, 1988; Kant, Fundamentación de la Metafísca de las costumbres, 1999; Kant, La metafísica de las costumbres, 1989). En su línea y su mejor exposición en Rawls y en su vertiente liberal en Dworkin (Rawls, Teoría de la justicia, 2006[1971]; Dworkin, El imperio de la justicia, 1992; Dworkin, Los derechos en serio, 1989; Dworkin, ¿Casos difíciles?, 1981).

${ }^{3}$ La línea liberal histórica tiene su raíz en Hegel (Hegel G. F., 2004; Hegel G. W., 1979). $\mathrm{Y}$ en sus representantes contemporáneos entre muchos se puede visualizar en la obra de Taylor (Taylor, La libertad de los modernos, 2005[1997]; Taylor, ¿Cuál es el problema de la libertad negativa?, 2005[1997]; Taylor, El atomismo, 2005[1997]). Habermas representa el desarrollo de la idea histórica de la justicia en la perspectiva de la Acción Comunicativa (Habermas \& Rawls, 1998; Habermas J. , Droit et démocracie, 1997; Habermas J. , Droit et démocratie. Entre faits et normes, 1992 ; Habermas J. , 1987) y entre muchos (Weinstock, 2001).

${ }^{4}$ (Sen , La idea de la justicia,, 2010; Sen, Bienestar, Justicia y mercado, 1997; Sen, Commodities and capabilities, 2000b; Sen, Nuevo examen de la desigualdad, 1995; Sen, Desarrollo y libertad, 2000)

${ }^{5}$ (Ayer, et. al (Comps), 1986) (Friedman, 1986) (Pareto, 1935) (Nozick, 1988) (Stegmüller, 1979) (Bolívar Espinoza G. A., 2010) (Bolívar Espinoza \& Flores, 2011) 


\section{Bibliografía}

Ayer, et. al (Comps). (1986), El positivismo lógico. Fondo de Cultura Económica. México.

Balzer, W. (1997), Teorías empíricas: modelos estructuras y ejemplos. Alianza. Madrid.

Balzer, W., Moulines, U., \& Sneed, J. (1987), An Architectonic for Sciences: The Structuralist Program. Reidel Publishing Company. Dordrecht.

Barry, B. (1997), La justicia como imparcialidad. Barcelona: Paidós.

Ídem (1993), La teoría liberal de la justicia. Fondo de Cultura Económica. México.

Ídem (1995[1989]), Teorías de la justicia. Gedisa. Barcelona.

Bolívar Espinoza, G. A. (2010), Un instrument d'analyse des reformes de l'État: les politiques institutionnelles de côntrole: l'Exaple mexicain (19821997). Éditions universitaires europeennes. Paris.

Bolívar Espinoza, G., \& Flores, L. (2011), “Loa avatares del capital social en América latina en Estancamiento, desigualdad y pobreza”. Documentación social, Revista de Estudios sociales y de Sociología Aplicada. Núm. 162 julio-septiembre, 261-293.

Campbell, T. (2002), La justicia. Gedisa. Barcelona.

De Lara, P. (2005[1997]), “La antropología filosófica de Charles Taylor”. En C. Taylor, La libertad de los modernos (págs. 13-29). Buenos AiresMadrid: Amorrortu/editores.

Diederich, W., \& Fulda, H. F. (1981), Estructuras sneedianas en el Capital de Marx. UNAM-Instituto de investigaciones filosóficas. México.

Dworkin, R. (1981), “¿Casos difíciles?” Cuadernos de Crítica Núm. 14, 582.

Ídem (1989), Los derechos en serio. Barcelona. Ariel.

Ídem (1992), El imperio de la justicia. Gedisa. Barcelona.

Friedman, M. (1986), “La metodología de la economía positiva”. En F. Hahn, \& M. Hollis, Filosofía y teoría económica (págs. 41-76). Fondo de Cultura Económica. México.

Habermas, J. (1987), Théorie de l'agir communicationnel. Fayard. Paris. 
Polis, Revista Latinoamericana, Volumen 14, $N^{\circ}$ 40, 2015

Ídem (1992 ), Droit et démocratie. Entre faits et normes. Gallimard. Paris. Ídem (1997), Droit et démocracie. Gallimard. Paris.

Habermas, J., \& Rawls, J. (1998), Debate sobre el Liberalismo Político. Paidós. Barcelona.

Hegel, G. F. (2004), La filosofía del derecho. Juan Pablo. México.

Ídem (1979), Encyclopédie des sciences philosophiques. I La sciences de ña logique . Librairie philosophique J. VRIN. Paris.

Kant, I. (1988), Crítica de la razón pura. Aguilar, AlteaTaurus, Alfaguara S. A., Santafé de Bogotá, Colombia.

Ídem (1989), La metafísica de las costumbres. (A. Cortina, J. Conill, Edits., A. Cortina, \& J. Conill, Trads.) Tecnos. Madrid.

Ídem (1999), Crítica de la razón práctica (Primera ed.). (D. M. Granja Castro, Ed., \& D. M. Granja Castro, Trad.) UAM-Iztapala. México.

Ídem (1999), Fundamentación de la Metafísca de las costumbres. Ariel. Barcelona.

Leriche, C., \& Caloca, Ó. (2009), Racionalidad y cooperación: un juego reflexico. Análisis Económico, 229-250.

Nozick, R. (1988), Anarchie, État et utopie. (trad. E. d'Auzac de Lamartine). P.U.F. Paris.

Pareto, V. (1935), The mind and society. Cape. London.

Rawls, J. (1995), Liberalismo político. Fondo de Cultura Económica. México.

Ídem (2006[1971]), Teoría de la justicia. Fondo de Cultura Económica. México.

Ídem (2007[2000]), Lecciones sobre la historia de la filosofía moral. Paidós. Barcelona.

Sen, A. (2010), La idea de la justicia. Taurus. México.

Ídem (1995), Nuevo examen de la desigualdad. Alianza. Madrid.

Ídem (1997), Bienestar, Justicia y mercado. Paidós. Barcelona.

Ídem (2000), Desarrollo y libertad. Planeta. México. 
Ídem (2000b), Commodities and capabilities. Oxford India. Delhi.

Stegmüller, W. (1979), Teoría y experiencia. Ariel. Barcelona.

Taylor, C. (2005[1997]), “¿Cuál es el problema de la libertad negativa?”. En C. Taylor, La libertad de los modernos (págs. 257-282). Amorrotu/editores. Buenos Aires-Madrid.

Ídem (2005[1997]), “El atomismo”. En C. Taylor, La lbertad de los modernos (págs. 225-256). Amorrotu/editores. Buenos Aires-Madrid.

Ídem (2005[1997]), La libertad de los modernos. Amorrotu/editores. Buenos Aires-Madrid.

Weinstock, D. (2001), Philosophie politique. S.N. ’Université de Montréalet et à l’Université Princeton.

Recibido: 28.07.2013

Aceptado: 30.06.2014 